\title{
PERFIL DOS PACIENTES ATENDIDOS NA ÁREA DE DISFUNÇÕES MUSCULOESQUELÉTICAS EM UMA CLÍNICA- ESCOLA DE FISIOTERAPIA DO NORTE DO RS
}

\section{Profile of patients cared for in the area of musculoskeletal dysfunctions in a physiotherapy school in northern rs}

\author{
Ivan Neves Evangelista Junior'; Fernanda Dal'Maso Camera²; Márcia Bairros de Castro²; \\ Janesca Mansur Guedes ${ }^{2}$
}

${ }^{1}$ Fisioterapeuta. Graduado pela URI Erechim.

${ }^{2}$ Fisioterapeuta. Doutora em Ciências da Saúde pela UNESC. Docente do Curso de Fisioterapia da URI Erechim. E-mail: janescaguedes@yahoo.com.br

RESUMO: As universidades apresentam um papel importante na sociedade, oferecendo serviços de qualidade nas mais diversas áreas de atendimentos à população. Os estudantes também são beneficiados por vivenciarem a prática antes de entrar no mercado de trabalho. Entre as diversas áreas oferecidas, a fisioterapia nas disfunções musculoesqueléticas é uma delas, a qual compreende as doenças do sistema locomotor e do tecido conectivo, que são a causa mais comum de incapacidade crônica no mundo. O objetivo do estudo foi descrever o perfil dos pacientes atendidos em uma Clínica-Escola de Fisioterapia na área de Disfunções Musculoesqueléticas (FDME). Foram analisados todos os prontuários de 2010 a 2017 na área do estágio supervisionado de FDME. Os dados verificados foram: gênero, ano da avaliação, idade, escolaridade, estado civil, região acometida, queixa principal, diagnóstico clínico, número de sessões realizadas. Os dados foram analisados com média, desvio padrão e frequência. $\mathrm{O}$ perfil dos pacientes que buscaram atendimento fisioterapêutico é predominantemente do sexo feminino, casado, com média de idade de 47 anos, apresentando o ensino médio completo, com queixa principal de dor na região lombar (Lombociatalgia), seguido do ombro (Síndrome do Impacto do Ombro) e joelho (Osteoartrose). Os pacientes realizaram uma média de 13 sessões de fisioterapia.

Palavras-chave: Epidemiologia. Fisioterapia. Disfunções Musculoesqueléticas. 
ABSTRACT: Universities play an important role in society, offering quality services in the most diverse areas of assistance to the population. Students also benefit from experiencing the practice before entering the job market. Among the various areas offered, physiotherapy for musculoskeletal disorders is one of them, which includes diseases of the locomotor system and connective tissue, which are the most common cause of chronic disability in the world. The aim of the study was to describe the profile of patients treated at a Physiotherapy School-Clinic in the area of Musculoskeletal Dysfunctions (MSDs). All medical records from 2010 to 2017 in the area of supervised MSDs internship were analyzed. The verified data were: gender, year of evaluation, age, education, marital status, affected region, main complaint, clinical diagnosis, number of sessions performed. Data were analyzed with mean, standard deviation and frequency. The profile of the patients who sought for physical therapy is predominantly female, married, with a mean age of 47 years old, holding complete high school education, with main complaint of low back pain, followed by the shoulder and knee, and who had an average of 13 physical therapy sessions.

Keywords: Epidemiology. Physiotherapy. Musculoskeletal Dysfunctions.

\section{Introdução}

As disfunções musculoesqueléticas compreendem as doenças do sistema locomotor e do tecido conectivo e são as causas mais comuns de incapacidade crônica no mundo. Algumas são predominantemente genéticas, enquanto outras dependem da associação de fatores genéticos e ambientais, associados a idade e/ou estão sob influência de fatores biológicos e psicossociais (GHISLENI, et al., 2014). O impacto destas condições sobre a economia é bastante significativo, não apenas em razão dos gastos diretos gerados pelo tratamento demandado, mas também pela consequente redução na produtividade. Com isso, ocorre o comprometimento de aspectos sociais e emocionais, além do bem-estar físico individual (WOOLF, et al., 2012).

O conhecimento da epidemiologia é de extrema importância para todas as áreas de atuação médica, uma vez que relaciona e fundamenta fatores encontrados até os dias atuais, podendo contribuir com tratamento e prevenção. Os estudos que abordam a fisioterapia contribuem com efeitos das técnicas, achados clínicos dos pacientes e aspectos epidemiológicos. (RODRIGUES, et al., 2017).

Dessa maneira, o presente estudo teve como objetivo geral conhecer o perfil dos pacientes atendidos na área de disfunções musculoesquelética (DME) na Clínica-Escola de Fisioterapia da Universidade Regional Integrada do Alto Uruguai e das Missões, URI Erechim, RS, e como objetivos específicos conhecer gênero, idade, escolaridade, estado civil; identificar a queixa principal, o diagnóstico clínico e região mais acometida; verificar o número de sessões oferecidas para os usuários da Clínica-Escola de Fisioterapia na área de DME.

\section{Metodologia}

O presente estudo caracteriza-se como descritivo, quantitativo e retrospectivo. Foram analisados 649 prontuários, foram inclu- 
PERFIL DOS PACIENTES ATENDIDOS NA ÁREA DE DISFUNÇÕES MUSCULOESQUELÉTICAS EM UMA CLÍNICA-ESCOLA DE FISIOTERAPIA DO NORTE DO RS

sos os prontuários completos dos pacientes atendidos na Clínica-Escola de Fisioterapia da URI Erechim, RS, na área de DME, entre os anos de 2010 e 2017. Os dados verificados foram: gênero, ano da avaliação, idade, escolaridade, estado civil, cidade, região acometida, queixa principal, diagnóstico clínico, número de sessões realizadas, percepção do estado geral de saúde e profissão.

Os dados obtidos foram tabulados em planilhas do software Microsoft Excel, aplicando estatística descritiva (média, desvio padrão, porcentagem) para a sua análise. Foi aprovado pelo Comitê de Ética e Pesquisa da URI de Erechim com o número 14858619.7.0000.5351.

\section{Resultados e Discussão}

Foram analisados 649 prontuários de pacientes atendidos na Clínica-Escola de Fisioterapia da URI Erechim, mediante atividades práticas no estágio curricular supervisionado de Fisioterapia nas Disfunções Musculoesquelética (DME): a idade média foi de 47,3 \pm 18 anos, com idade mínima de quatro anos e idade máxima de 87 anos, a faixa etária com maior prevalência foi entre 51 a 60 anos (22,8\%) (Tabela I), e o gênero feminino foi predominante, com $66 \%$ (429) e o gênero masculino com $34 \%$ (220).

Com base nos dados da amostra estudada, observa-se a faixa etária com maior prevalência nos atendimentos como sendo entre 51 a 60 anos de idade, seguida por 41 a 50 anos e 61 a 70 anos de idade.

Os resultados deste estudo em relação à idade e ao gênero dos pacientes corroboram a pesquisa de Oliveira e Braga (2010), que verificou o perfil epidemiológico de pacientes atendidos na clínica de ortopedia da Universidade Paulista. Em relação ao gênero, obser-va-se que os homens têm uma resistên- cia maior ao buscar auxílio à saúde (SOUZA et al., 2011; GHISLENI et al., 2014).

Tabela I - Categorização da faixa etária da amostra estudada

\begin{tabular}{c|cc}
\hline VARIÁVEIS & $\mathrm{N}$ & $\%$ \\
\hline $0-10$ & 7 & 1,1 \\
$11-20$ & 50 & 7,7 \\
$21-30$ & 76 & 11,6 \\
$31-40$ & 82 & 12,6 \\
$41-50$ & 132 & 20,4 \\
$51-60$ & 148 & 22,8 \\
$61-70$ & 88 & 13,6 \\
$71-80$ & 52 & 8,0 \\
$81-90$ & 14 & 2,2 \\
\hline Total & 649 & 100 \\
\hline
\end{tabular}

No presente estudo, os pacientes realizaram uma média de $13,1 \pm 5,8$ sessões de fisioterapia por paciente, ao total foram oferecidas 8.508 sessões no período de 2010 a 2017 na área de Estágio Curricular Supervisionado de Fisioterapia nas Disfunções Musculoesqueléticas.

A média de medicamentos utilizados pelos pacientes foi de $2,2 \pm 2,1$, com um mínimo de zero e máximo de 11 medicamentos por pessoa. Quanto à percepção de saúde, observa-se que 249 (38,4\%) pacientes atendidos relatam ter uma boa saúde, 233 $(35,9 \%)$ ter uma saúde regular, $73(11,2 \%)$ uma saúde ruim, 46 (7\%) uma saúde muito boa e 21 (3\%) uma saúde muito ruim.

De acordo com os prontuários analisados, $614(94,3 \%)$ dos pacientes atendidos eram do município de Erechim, os demais municípios encontrados nesta pesquisa correspondem à região norte do Alto Uruguai, com pacientes de 15 municípios, entre os quais nove $(1,4 \%)$ pacientes de Gaurama e seis $(0,9 \%)$ pacientes de Getúlio Vargas.

O grau de instrução da amostra foi categorizado entre 5 classes, sendo elas: Analfabeto, 
Ensino Fundamental Incompleto, Ensino Fundamental Completo (encontra-se a classe do Ensino Médio Incompleto), Ensino Médio Completo (onde se encontra a classe do Ensino Superior Incompleto) e Ensino Superior Completo (Tabela II). Neste estudo verificou-se que a maioria dos pacientes apresentaram $2^{\circ}$ grau completo.

Tabela II - Grau de Instrução da amostra estudada

\begin{tabular}{c|cc}
\hline VARIÁVEIS & $\mathrm{n}$ & $\%$ \\
\hline Analfabeto & 8 & 1 \\
$\begin{array}{c}\text { Ensino Fundamental } \\
\text { Incompleto } \\
\text { Ensino Fundamental } \\
\text { Completo }\end{array}$ & 196 & 32 \\
$\begin{array}{c}\text { Ensino Médio } \\
\text { Completo } \\
\text { Ensino Superior } \\
\text { Completo }\end{array}$ & 99 & 10 \\
\hline Total & 73 & 41 \\
\hline
\end{tabular}

Quanto ao grau de instrução, podemos verificar que a escolaridade mais encontrada foi o Ensino Médio Completo, com 39\% da amostra pesquisada.

Em relação ao estado civil, a amostra foi classificada em casado, solteiro, divorciado e viúvo, e verificou-se uma prevalência de $344(54 \%)$ de pessoas casadas, $182(28 \%)$ de solteiras, 70 (11\%) divorciadas e 45 (7\%) viúvas. A queixa principal mais relatada no presente estudo foi de dor, com 610 (60\%), seguida de dificuldade em realizar as atividades de vida diária $352(34,5 \%)$ e, finalmente, a perda de função do segmento afetado, com $56(5,5 \%)$. Destacando que alguns pacientes apresentavam mais de uma queixa.

Assim como no estudo (DA-SILVA; et al, 2015), a incidência da dor é a mais prevalente, entre as queixas dos pacientes.

As profissões de diarista $(8,9 \%)$, estudante $(5,5 \%)$, agricultor $(3,5 \%)$ e costureira $(2,6 \%)$ foram as mais relatadas, no entanto
453 prontuários não estavam completos em relação a esta pergunta.

Referente ao diagnóstico clínico, o mais prevalente foi a lombociatalgia com 86 casos $(12,4 \%)$ e, em seguida, a síndrome do impacto do ombro (SIO) com 78 pacientes $(11,4 \%)$. Outros diagnósticos como lombalgia, fraturas e osteoartrose de joelho também foram significativos (Tabela III).

Tabela III - Principais patologias em evidência na amostra estudada

\begin{tabular}{l|cc}
\hline VARIÁVEIS & $\mathrm{n}$ & $\%$ \\
\hline Síndrome Fêmoro-Patelar & 8 & 1,1 \\
Pós-Operatório LCA & 10 & 1,4 \\
Artrodese de Coluna & 11 & 1,4 \\
Epicondilite Lateral & 13 & 1,9 \\
Entorse de Tornozelo & 14 & 1,9 \\
Prótese Total de Quadril & 14 & 2 \\
Cervicobraquialgia & 15 & 2,2 \\
Pós-Operatório de Manguito & 20 & 2,7 \\
Rotador & 23 & 3,4 \\
Prótese Total de Joelho & 26 & 3,7 \\
Cervicalgia & 26 & 3,7 \\
Escoliose & 29 & 4 \\
Osteoartrose de Joelho & 40 & 4,1 \\
Fratura de Membro Superior & 44 & 5,1 \\
Fratura de Membro Inferior & 50 & 7,2 \\
Lombalgia & & \\
Síndrome do Impacto do & 78 & 11,4 \\
Ombro & 86 & 12,4 \\
Lombociatalgia & 105 & 22,1 \\
Outras & 683 & 100 \\
\hline Total & & \\
\hline & & \\
\hline
\end{tabular}

Em relação ao diagnóstico clínico, somando a incidência de lombalgia e lombociatalgia corresponde a 146 pacientes atendidos, $19,6 \%$ do total das patologias, o que vem ao encontro da literatura que diz que a lombalgia é tão comum na idade adulta que estudos documentaram a prevalência de lombalgia ao longo da vida chegando aos 80\%. (ANDREWS; HARRELSON; WILK, 2005). 
De acordo com os prontuários analisados, a maioria dos pacientes apresenta a coluna lombar $(12,4 \%)$ como segmento mais acometido, seguido do ombro (11,4\%) e o joelho $(4 \%)$.

Comparando os dados do diagnóstico clínico, com o segmento corporal acometido, confirmamos os dados, sendo que o diagnóstico clínico mais prevalente de lombociatalgia corrobora o segmento corporal mais acometido, que é a coluna lombar.

\section{Conclusão}

Conclui-se que a maioria dos pacientes atendidos na Clínica-Escola de Fisioterapia da URI Erechim, na área de Disfunções Musculoesqueléticas no período de 2010 a 2017, são do sexo feminino, adultos, casados, buscam atendimento por dor na região lombar e que realizaram uma média de treze sessões de fisioterapia.

\section{REFERÊNCIAS}

ANDREWS, J. R.; HARRELSON, G. L.; WILK, K. E. Reabilitação Física do Atleta. 3. ed. Rio de Janeiro: Elsevier, 2005.

CARIOLANDO DA SILVA, K. D. O.; et al. Perfil dos Pacientes Atendidos na Clínica-Escola de Fisioterapia no Setor de Ortopedia e Traumatologia. Revista Eletrônica Estácio Saúde, v. 4, n. 1, p. $1-7,2015$.

FERREIRA, A. H. et al. Investigação da ansiedade, depressão e qualidade de vida em pacientes portadores de osteoartrite no joelho: um estudo comparativo. Revista Brasileira de Reumatologia, v. 55, n. 5, p. 434-438 2015.

GHISLENI, M.M. et al. Perfil epidemiológico dos pacientes atendidos na área de ortopedia e traumatologia da clínica-escola de fisioterapia Univates. Revista destaques acadêmicos, v. 6, n. 3, p. 1-9, 2014.

GARCIA FILHO, R., J., et al., Ensaio Clínico Randomizado, Duplo-cego, Comparativo Entre a Associação de Cafeína, Carisoprodol, Diclofenaco Sódico e Paracetamol e a Ciclobenzaprina, para Avalização da Eficácia e Segurança no Tratamento de Pacientes com Lombalgia e Lombociatalgia Agudas. Acta Ortopédica Brasileira, v. 14, n. 1, p. 11-16, 2006.

LIMA, G., C. S. et al., Análise da funcionalidade e da dor de indivíduos portadores de síndrome do impacto, submetidos à intervenção fisioterapêutica. Revista Fisioterapia em Movimento, v. 20, n. 1, p. 61-69, 2007.

METZKER, C., A. B. Tratamento conservador na síndrome do impacto no ombro.

Revista Fisioterapia em Movimento, v. 23, n. 1, p. 141-151, 2010.

MICHENER, L. A. et al. Anatomical and Biomecanical mechanisms of subcondral impingement syndrome. Clinical Biomechancs, v. 18, n. 5, p. 369-379, 2013.

OLIVEIRA JUNIOR, J., OSWALDO; LAGES, G., V. Ozonioterapia em lombociatalgia. Central da Dor e Estereotaxia do Hospital Antonio Cândido Camargo da Fundação Antonio Prudente (FAP).

Revista Dor, v. 13, n. 3. p. 261-70, 2012.

PRENTICE, W. E. Fisioterapia na Prática Esportiva: Uma abordagem baseada em competências. 14. ed. Porto Alegre: AMGH, 2012. 
POZZI, J. F. A.; KONKEWICZ, E. R. Joelho do Adulto. In.: HEBERT, SIZÍNIO et al. Ortopedia e Traumatologia: Princípios e Prática, 3. ed. Porto Alegre: Artmed, 2003.

RODRIGUES, W., C. C. et al., Perfil epidemiológico e clínico de pacientes com queimaduras atendidos pela fisioterapia na Universidade Estadual de Goiás. Revista Brasileira de Queimaduras, v. 16, n. 2, p. 94-99, 2017.

UMER, M. et al. Subacromial Impingement Syndrome. Orthopedic Reviews, v. 4, n. 18, p. 79-82, 2012.

WOOLF, A.D. et al., The need to address the burden of musculoeskeletal conditions. Best Practice and Research Clinical Rheumathology, v. 26, n. 2, p. 183-224, 2012. 